\title{
ANÁLISE DO RELACIONAMENTO ENTRE A GESTÃO DO CONHECIMENTO E AS PRÁTICAS PARA MELHORIA DA QUALIDADE: ESTUDO DE CASO EM UMA EMPRESA DE ALTA TECNOLOGIA
}

\author{
ANALYSIS OF THE RELATIONSHIP BETWEEN KNOWLEDGE \\ MANAGEMENT AND PRACTICES OF QUALITY IMPROVEMENT: \\ STUDY OF CASE IN A COMPANY OF HIGH TECHNOLOGY
}

\author{
Claudia Andressa Cruz ${ }^{1}$; Edna de Almeida Rodrigues ${ }^{2}$; Marcelo Seido Nagano $^{3}$ \\ ${ }^{1}$ University of São Paulo - USP - São Carlos - Brasil claudia.andressa@gmail.com \\ ${ }^{2}$ University of São Paulo - USP - São Carlos - Brasil rodrigues.pmep@ig.com.br \\ ${ }^{3}$ University of São Paulo - USP - São Carlos - Brasil drnagano@usp.br
}

\begin{abstract}
Resumo
Este artigo pretende evidenciar a relação existente entre gestão do conhecimento e as práticas de qualidade a fim de comprovar como está caracterizado todo o processo de gestão do conhecimento anterior à implantação de técnicas de qualidade. O trabalho foi desenvolvido através de um estudo de caso em profundidade cuja coleta de dados ocorreu por meio de entrevista semi-estruturada aplicada em uma empresa integrante ao parque tecnológico da cidade de São Carlos/SP.
\end{abstract}

Palavras-chave: gestão do conhecimento; qualidade, parque tecnológico.

\section{Introdução}

As abordagens contemporâneas afirmam que proporcionar valor junto ao cliente é um meio de conquistar posições privilegiadas no mercado em que a empresa está inserida. Por um lado, as mudanças ambientais dos últimos tempos possibilitam melhorias no desenvolvimento organizacional, e, por outro, demandam mudanças radicais por parte dos gestores e dos governos. Esta é uma das preocupações da gestão do conhecimento e das práticas de qualidade que buscam a satisfação do cliente. O conhecimento é inerente ao ser humano e disseminá-lo pela organização garante a sua continuidade, pois é através da gestão do conhecimento que a empresa pode conquistar vantagem competitiva. Qualidade, por sua vez, envolve práticas para a satisfação total do cliente de forma a proporcionar à empresa um posicionamento seguro em tempos de mercados competitivos.

O avanço cientifíco-tecnológico impôs novos padrões de desenvolvimento econômico, em que a automação e a flexibilização do processo produtivo provocaram alterações nas economias de 
escala cuja aqueles que investiram em ciência e tecnologia obtiveram posição de destaque. Internamente, foi necessário estruturar novas parcerias e aproximar pesquisador e empreendedor. Uma das alternativas foi a formação de parques tecnológicos, que reúnem empresas próximo ao campus da universidade. Devido às características peculiares dos parques tecnológicos, este estudo pretende contribuir com o aprimoramento dos estudos sobre gestão do conhecimento e práticas de qualidade neste ambiente.

\section{Gestão do conhecimento}

Em gestão do conhecimento, o principal capital organizacional é o humano, o único responsável pela criação do conhecimento. A organização deve ser capaz de captar, armazenar e transmitir o novo conhecimento organizacional criado a partir da transformação do conhecimento tácito interno, individual - em conhecimento explícito - técnico, formalizado. Ainda é necessária a adequação da estrutura organizacional para permitir e incentivar a criação de novas idéias (SVEIBY, 1998; NONAKA e TAKEUCHI, 1997; DAVENPORT e PRUSAK, 2003).

Conforme Sveiby (1998), as pessoas geram idéias, interagem e criam nas organizações as estruturas interna e externa para efetivar essas expansões direcionando seus esforços para fora da organização trabalhando com clientes e fornecedores.

Segundo Nonaka e Takeuchi (1997), a criação do conhecimento é a interação contínua e dinâmica entre o conhecimento tácito e o explícito, conforme ilustrado na figura 1.

Figura 1: Espiral do conhecimento

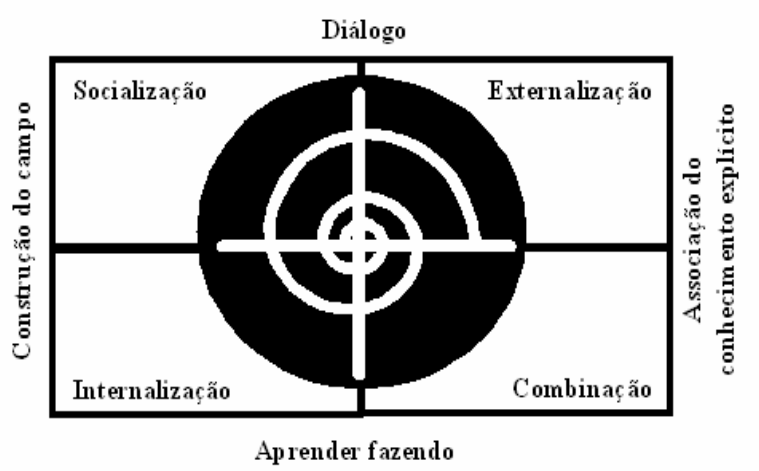

Fonte: adaptado de Nonaka e Takeuchi (1997)

A socialização é o processo de compartilhamento de experiências que cria conhecimento tácito, o qual é articulado em conhecimento explícito na externalização através do diálogo. A combinação é o processo de sistematização do conhecimento explícito em sistema de conhecimento onde os indivíduos trocam e combinam conhecimento que é incorporado na internalização através do “aprender fazendo". Quando a maioria compartilha do novo modelo mental, o conhecimento tácito passa a fazer parte da cultura organizacional e a cada internalização bem sucedida, o ciclo reinicia, levando ao aperfeiçoamento ou à inovação. 
Figura 2: Representação conceitual de $b a$

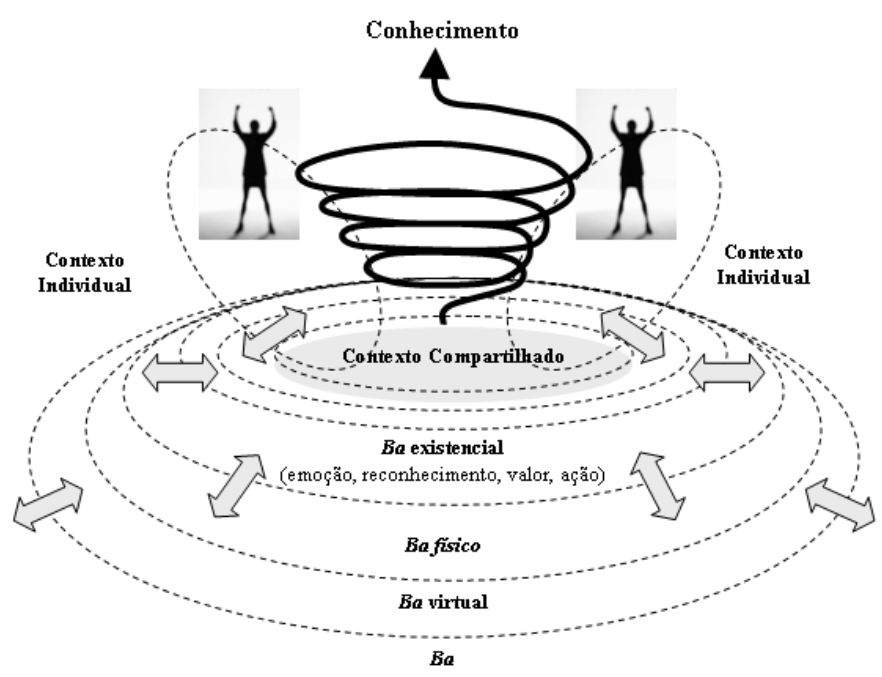

Fonte: adaptado de Nonaka e Toyama (2003)

Nonaka e Toyama (2003) introduzem o conceito ba como um ambiente para a criação de conhecimento organizacional representado como um local onde o conhecimento é continuamente compartilhado, criado e utilizado pelos indivíduos, conforme figura 2. A empresa, como organismo, possui vários $b a$ 's que possibilita evidenciar o tipo de conhecimento a ser criado, identificar as pessoas com conhecimento embutido e verificar a forma de interação adequada para criação de conhecimento sem as restrições da estrutura organizacional.

\section{Práticas de qualidade}

O termo qualidade vem sendo analisado sob várias abordagens distintas, originando inúmeros conceitos e definições. Em uma linguagem técnica, o termo "qualidade" assume definições cujos significados e abrangências também podem corresponder à filosofia de gestão empresarial. Conforme alguns autores, qualidade pode significar: adequação ao uso; conformidade com requisitos; ainda uma combinação de características de produtos e serviços referentes a marketing, engenharia, produção e manutenção, através dos quais produtos e serviços em uso corresponderão às expectativas dos clientes. De acordo com Garvin (1992), quatro principais eras podem ser identificadas:

a) Inspeção: corresponde a um enfoque de verificação da conformidade do produto/serviço final e segregação das unidades não conformes;

b) Controle Estatístico do Processo: era em que a função reativa de inspeção passou para preventiva; segue a premissa de garantir uniformidade do produto/serviço através do controle das variáveis que podem influir na qualidade final do produto; 
c) Garantia e Controle da Qualidade: corresponde a mudança de foco da qualidade do produto final para a qualidade nas tarefas e processos diários;

d) Gestão da Qualidade Total: remete a uma visão global da organização como resultado do aumento da integração de diferentes aspectos funcionais da qualidade.

Alguns gerentes denominam a Gestão da Qualidade Total (TQM) como um novo modelo de pensamento, ou ainda como uma mudança de paradigma. Ao contrário de práticas passadas que eram orientadas pelo resultado, TQM é visto como uma filosofia orientada ao processo de valorização da satisfação do cliente.

Gianesi e Corrêa (1994) fundamentam o conceito de qualidade total da seguinte forma:

- Todos na organização têm algo a contribuir para a qualidade final percebida pelo cliente $[\ldots]$

- Todo o esforço bem direcionado de melhoria em qualidade repercute na competitividade [...];

- A qualidade deve ser construída ao longo do processo e não apenas verificada no final (GIANESI e CORREAA, 1994, p.197).

Em especial, as empresas produtoras de software passam pelo desafio da melhoria da qualidade. Segundo Sandhof (2004), a consciência de melhoria contínua, de definição de processos, de gestão, dentre outros atributos, pode levar uma empresa a passar a um nível de maturidade significativo independente do modelo de melhoria da qualidade adotado por ela. A qualidade de um software está baseada na tríade processo-tecnologia-pessoas e esta junção conduz a organização a um patamar de capacitação que eleva seus processos e produtos à qualidade desejada, conforme apresentado na figura 3.

O envolvimento de todos os funcionários da organização no processo de tomada de decisão e a qualidade de troca de informação aumenta consideravelmente, resultando em maiores índices de aprendizagem (SOARES JÚNIOR, 1996 apud IOMBRILLER, 1999, p.45).

Figura 3: Dimensões críticas da capacidade organizacional

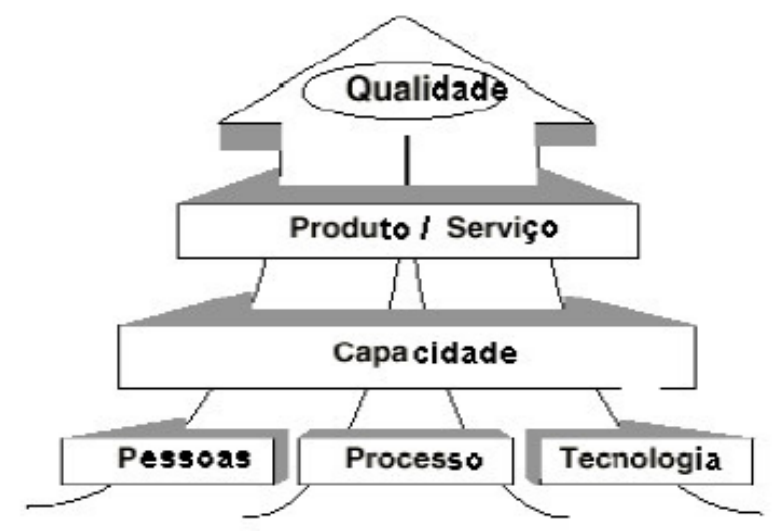

Fonte: adaptada de Armitage (1993 apud Sandhof, 2004)

Um conceito de marketing utilizado que prioriza uma filosofia focada nos clientes, é o CRM (Customer Relationship Management), isto é, Gestão de Relacionamentos de Clientes, que ${ }_{48}$ 
estabelece um conjunto de estratégias, processos e ferramentas para conhecer melhor os clientes, de modo a tornar possível a implementação de ações para satisfazê-los e fidelizá-los, expandindo as oportunidades de negócios (SILVA, 2002 apud VICENTE, 2003, p.7). Os objetivos principais do CRM são: permitir que a empresa identifique, contate e consiga novos clientes com maior eficiência, e aprimore o relacionamento da empresa com os clientes que já conquistou (CHERRYTREE, 2000 apud VICENTE, 2003, p.7).

$\mathrm{O}$ artigo apresenta nos próximos itens a metodologia empregada e o estudo de caso.

\section{Metodologia}

Dessa forma, a presente pesquisa busca investigar empiricamente a relação entre a gestão do conhecimento e as práticas de melhorias da qualidade, mediante um estudo em uma empresa de alta tecnologia. Interface esta, ainda, pouco explorada na literatura, o que caracteriza uma pesquisa exploratória e descritiva, seja por realizar descrições precisas da situação, visando descobrir as relações existentes entre os elementos componentes da mesma, bem como explorar um assunto pouco abordado, medindo suas características (DANE, 1990; HAIR Jr. et al., 2005).

Optou-se pelo delineamento estudo de caso em virtude dos seguintes aspectos: primeiramente, porque a problemática de pesquisa remete a um conjunto contemporâneo de acontecimentos sobre o qual a pesquisadora tem pouco controle; secundariamente, por investigar, a gestão do conhecimento, um fenômeno contemporâneo, em uma empresa de base tecnológica, dentro do contexto da vida real, em que os limites entre o fenômeno e o contexto não estão claramente definidos, tendo em vista a carência de literatura nacional sobre a temática (YIN, 2004).

Neste âmbito foi realizada uma entrevista realizada do tipo focal (MERTON et al.,1990 apud YIN, 2001), na qual o respondente foi entrevistado por um curto período de tempo, e embora a entrevista tenha sido espontânea, um certo conjunto de perguntas foi seguido.

\section{Apresentação da Pesquisa e Análise dos Resultados}

A pesquisa foi realizada conforme os seguintes procedimentos: escolha da amostra investigada; contato estreito e duradouro com a unidade de pesquisa; e investigação em profundidade, buscando captar e compreender suas especificidades. A entrevista buscou evidenciar o problema central, a partir das questões:

- Quais são os procedimentos/ferramentas utilizados que visam a melhoria da qualidade?

- O conhecimento criador de valor é externalizado pela organização?

- A gerência reconhece a relação da gestão do conhecimento com as práticas de qualidade?

\subsection{Estudo de Caso}


De acordo com Anprotec (2004), no Brasil há um total de 39 parques, dos quais 48,7\% estão localizados na região sudeste. A unidade estudada está inserida no Parque Tecnológico de São Carlos - Parqtec, uma instituição de direito privado, sem fins lucrativos, que foi criada em dezembro de 1984 pelo Conselho Nacional de Desenvolvimento Científico e Tecnológico -CNPQ e gerenciada por iniciativa da comunidade acadêmica local, além de contar com o apoio financeiro de entidades de fomento à pesquisa e tecnologia em nível nacional. Sua principal atividade é propiciar condições para a criação de empresas de alta tecnologia geradas no ambiente universitário. A escolha de uma empresa de base tecnológica remete ao fato de que representam $94 \%$ do total de parques tecnológicos instalados no país.

A unidade de análise foi criada há 5 anos e apresenta como missão desenvolver soluções de tecnologia voltadas para a gestão da operação logística. A empresa possui 8 funcionários e 5 terceirizados. Os principais clientes são: Alcoa, Brasilsat, Controlsat, Copagaz, Estrada Transportes, Omnitrans Logística, Supermix Comercial, Viação Santa Cruz, VCP.

\subsection{Análise dos resultados obtidos}

A apresentação dos resultados da pesquisa está dividida em três tópicos: práticas de qualidade; gestão do conhecimento e relação entre gestão do conhecimento. A análise foi feita procurando responder às questões do estudo.

\subsubsection{Práticas de qualidade}

Segundo a empresa, o conceito de qualidade pode ser visto sob dois ângulos: o primeiro referente à qualidade do produto e o segundo à qualidade do serviço. A qualidade do produto é o desenvolvimento de um software que atenda às especificações, isto é, atenda às funcionalidades propostas; enquanto a qualidade em serviço engloba suporte, tempo de resposta, tempo de correção do problema, pré-venda e atendimento.

As práticas para qualidade do produto são referentes a uma metodologia de desenvolvimento de produtos que foi adaptada de outras existentes, nas quais os produtos são desenvolvidos em etapas e agregações são feitas às novas funcionalidades. A cada vez que uma funcionalidade nova é agregada, eles lançam o chamado release, o qual passa por uma bateria de testes internos e externos. O teste interno analisa a função específica, ou o método, que é um trecho do software, este é analisado através da verificação da conformidade com as especificações. O teste externo é feito por uma pessoa que não participou do desenvolvimento, e a partir dos requisitos, a conformação do software é verificada.

Para a qualidade da prestação de serviço, a empresa registra em um CRM todos os eventos com 
os clientes, tais como: eventos de suporte, eventos de dúvidas, eventos de bugs, dentre outros. Mensalmente, um relatório é elaborado e são analisados todos os registros.

Uma abordagem adotada pela empresa, é a formação de equipes de projeto cuja quantidade de membros varia entre 2 a 8 pessoas de acordo com a complexidade do projeto. Estas equipes são caracterizadas como matriciais, isto é, são constituídas por equipes multi-disciplinares de projetos com vida finita, coordenando e planejando suas próprias atividades. A liderança da equipe é rotativa e a atividade principal do líder é a gestão do projeto, desde cronogramas, atividades, resultados até o acompanhamento dos testes. O líder raramente mantém relacionamento com o cliente, que é incumbência da gerência.

\subsubsection{Gestão do Conhecimento}

Gestão do conhecimento é compreendida como acumulação e disseminação dos conhecimentos técnico e organizacional. Os recursos utilizados pela empresa para disseminar o conhecimento interno são palestras, murais virtuais, intranet, MSN, e-mail, reuniões trimestrais, copa, happy hour e CRM, sendo que este último possui acesso restrito. No que concerne à disseminação cliente-empresa, é empregado o uso do site na internet, além de uma revista eletrônica semanal com notícias da área e da empresa. Em relação aos fornecedores não há relacionamento específico, pois estes fornecem tecnologia pronta, tais como: computadores, softwares que produzem software, e ambientes de software.

Para obter novos conhecimentos necessários aos negócios da organização, a empresa tem como característica investir em seus próprios funcionários. É comum que haja um livro relacionado às necessidades de atualização sobre as mesas de trabalho e a contratação de cursos para aprimoramento técnico.

A importância das atividades de Pesquisa e Desenvolvimento (P\&D) dentro do processo de criação de conhecimento está na utilização de uma tecnologia bastante recente, então é fundamental que a equipe de $\mathrm{P} \& D$ estude para atualização constante, caso contrário a empresa perde mercado. $\mathrm{O}$ gerente neste processo tem por função incentivar e fornecer os meios.

\subsubsection{Relação entre gestão do conhecimento e práticas de qualidade}

As parcerias com clientes são um dos principais elos evidenciados entre a gestão do conhecimento e a qualidade, pois a prioridade da empresa está no foco com o cliente, tudo é definido de acordo com as necessidades dele e há uma intensa captação desde conhecimento, a fim de identificar as especificações desejadas. Portanto, o conhecimento é criado a partir das necessidades dos clientes que as transferem a partir do seu conhecimento tácito, e explicitam-no através do cadastramento das necessidades no CRM. 
Este conjunto de estratégias, processos e ferramentas do CRM para conhecer melhor os clientes, é o principal provedor da criação do conhecimento do presente estudo de caso, pois a partir dele é possível a implementação de ações para satisfazer e fidelizar os clientes (SILVA, 2002 apud VICENTE, 2003).

Para manter um contato direto com os clientes e para identificar seus requisitos, há também um banco de dados de cada produto que é compartilhado com os clientes, que verificam através de seu conhecimento se há problemas nas especificações, o qual é externalizado e a empresa altera a nãoconformidade beneficiando todos os clientes.

As palestras e cursos proporcionados pela empresa à especialização de seus funcionários também é uma forma de criação do conhecimento, pois a partir deste incentivo ao aprendizado, melhorias podem ser obtidas nas práticas de qualidade.

\section{Considerações Finais}

No que concerne ao produto, é observado claramente a era de controle estatístico do processo em que há a premissa de garantir uniformidade do produto através do controle das variáveis que podem influir na qualidade final do produto, o que denota a visão de qualidade segundo Crosby (1990) de conformidade com requisitos. Em relação à qualidade do serviço, a visão que apresenta maior proximidade, seria a combinação de características de serviços referentes a marketing, engenharia, produção e manutenção, através dos quais os serviços em uso corresponderão às expectativas dos clientes. Assim, uma combinação de eras foi constatada, não permitindo uma visão clara sobre qual seriam as melhores práticas no que concerne a qualidade. Isto, provavelmente, é devido a recente fundação da empresa.

O gerente reconhece que as práticas de qualidade podem proporcionar melhorias de desempenho, pois após a agregação dos testes externos para verificação da conformidade, houve diminuição dos custos com retrabalhos e conseqüente aumento de produtividade. A empresa não consegue inferir diretamente quanto seria o percentual de melhoria após a implantação destes testes, pois outras tarefas paralelas a estas foram realizadas.

$\mathrm{Na}$ empresa predomina a visão técnica, pois foi observado que a qualidade dos softwares desenvolvidos está baseada mais na tecnologia empregada e no know how dos funcionários. Embora as habilidades técnicas sejam importantes, elas não devem ser priorizadas isoladamente. Assim, os autores deste estudo sugerem um investimento maior em outras necessidades que as pessoas, tanto clientes como funcionários, possam vir a ter, a fim de obter uma melhor composição das dimensões da capacidade organizacional, apoiada na tríade processo-tecnologia-pessoas para alcançar a qualidade dos produtos e serviços (ARMITAGE, 1993 apud SANDHOF, 2004). 
A gerência busca a uniformidade do conhecimento técnico como condição básica para ter uma equipe produtiva. Se o conhecimento é individual, essa busca pela uniformidade indica que a filosofia da empresa é priorizar o conhecimento teórico, expresso. Entretanto, para a boa gestão do conhecimento, conforme as definições de Nonaka e Takeuchi (1997) sobre conhecimento organizacional, a empresa deve priorizar o conhecimento tácito, individual, carregado com vivências e experiências para então enriquecer aquele conhecimento técnico.

As respostas do questionário foram analisadas procurando identificar a solução da questão central - evidenciar a relação entre a gestão do conhecimento e as práticas para melhoria da qualidade -, conforme demonstrado na figura 4.

Figura 4: Respostas das questões da pesquisa

\begin{tabular}{|c|l|}
\hline \hline \multicolumn{1}{|c|}{ Questão central da pesquisa } & \multicolumn{1}{c|}{ Resposta extraída da pesquisa } \\
\hline \hline $\begin{array}{c}\text { A gestão do conhecimento é parte intrínseca das } \\
\text { práticas de qualidade? }\end{array}$ & $\begin{array}{c}\text { Sim, porque as pessoas são treinadas para suas } \\
\text { atividades, embora seja um processo desestruturado. }\end{array}$ \\
\hline \hline Questões secundárias da pesquisa & \multicolumn{1}{c|}{ Respostas extraídas da pesquisa } \\
\hline \hline $\begin{array}{l}\text { Quais são os procedimentos/ferramentas } \\
\text { utilizados que visam a melhoria da qualidade? }\end{array}$ & $\begin{array}{l}\text { Os principais são: CRM, testes internos e externos de } \\
\text { conformidade, suporte técnico e acompanhamento dos } \\
\text { níveis de satisfação do cliente. }\end{array}$ \\
\hline $\begin{array}{l}\text { O conhecimento criador de valor é } \\
\text { externalizado pela organização? }\end{array}$ & $\begin{array}{l}\text { Não, pois há priorização do conhecimento técnico em } \\
\text { detrimento do relacionamento criador de conhecimento. }\end{array}$ \\
\hline 3. $\begin{array}{l}\text { A gerência reconhece a relação da gestão do } \\
\text { conhecimento com as práticas de qualidade? }\end{array}$ & $\begin{array}{l}\text { Não totalmente, porque o conhecimento tácito encontra } \\
\text { pouco espaço de interação e a qualidade é vista apenas sob } \\
\text { o ponto de vista técnico. }\end{array}$ \\
\hline
\end{tabular}

Através do estudo de caso foi possível construir a figura 5, que representa a forma como ocorre a criação do conhecimento na empresa pesquisada, atualmente. O conceito de "espiral do conhecimento" sistematizado por Nonaka e Takeuchi (1997) serviu como orientador destas considerações pois é através da disseminação do conhecimento em todos os sentidos, em todos os níveis e para dentro e para fora da organização que o novo conhecimento é criado. Na figura, a espiral está representada por linha pontilhada, devido ao processo desestruturado de gestão do conhecimento encontrado na empresa.

Conforme Nonaka e Toyama (2003), o processo de criação de conhecimento necessita de um $b a$, local onde a informação ganha significado através da interpretação para ser transformada em conhecimento. Na empresa analisada, a gestão do conhecimento é base para práticas de melhoria, sendo que técnicas como: CRM, cursos, palestras e a dinâmica interação com o ambiente, são utilizadas para que o conhecimento criado resulte em melhorias das práticas de qualidade para satisfação total do cliente. 
Figura 5: A espiral do conhecimento de Nonaka e Takeuchi e a influência da gestão de conhecimento sobre práticas de melhoria da qualidade em uma empresa de base tecnológica

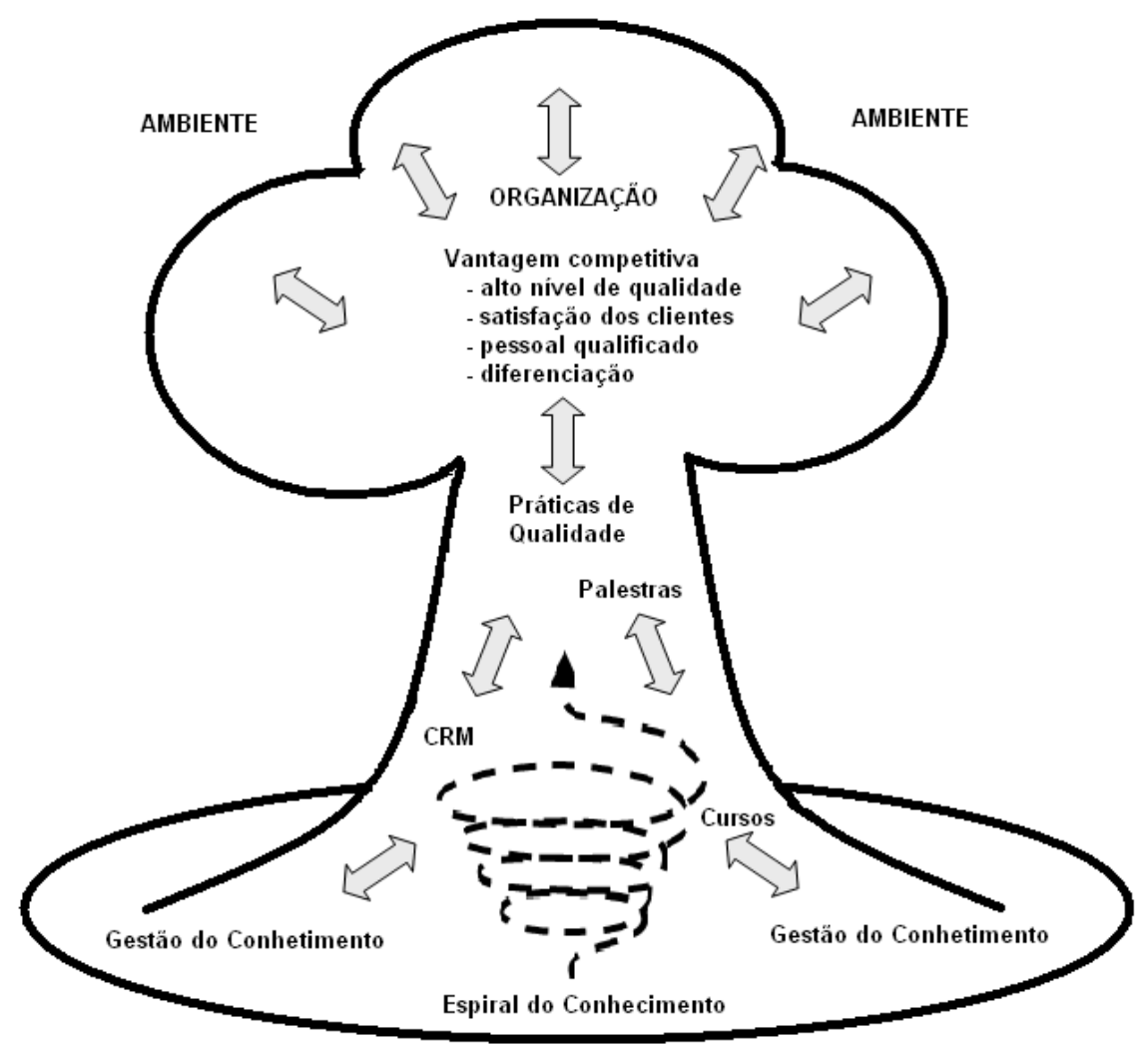

Os autores identificaram como delimitações da pesquisa a inadequação da teoria sobre qualidade à organização de alta de tecnologia, considerando as características peculiares da atividade. Portanto, sugerem que futuras pesquisas busquem explorar essas especificidades.

\section{Abstract}

The present work accomplishes an analysis of the existing relation between Knowledge Management (KM) and Quality's Practices in a high technology firm. For that, the knowledge management process had been evidenced previous quality's practices through interview semistructure applied in an integrant firm to the science park, in order to do an evaluation of the interface knowledge management and quality could be accomplished. Finally, this study proposes improvements to the existing practices and suggests some alternatives that aim maximize the dissemination and creation of the knowledge in the studied firm.

Key words: Knowledge management; Quality; High technology firm.

\section{Referências}

PANORAMA 2004, Brasília: Associação Nacional de Entidades Promotoras de Empreendimentos Inovadores ANPROTEC. 2004. 
CROSBY, P. B. Qualidade: falando sério. São Paulo: McGraw-Hill. 1990.

DANE, F.C.. Research methods. Belmont: Brooks/Cole. 1990.

DAVENPORT, T. H.; PRUSAK, P. Conhecimento empresarial: como as organizações gerenciam o seu capital intelectual. Título original: Working Knowledge, tradução de Lenke Peres, Rio de Janeiro: Elsevier, p.6. 2003.

GARVIN, D. A. Gerenciando a qualidade: a visão estratégica competitiva. Rio de Janeiro: Qualitymark. 1992.

GIANESI, I. G. N.; CORRÊA, H. L. Administração estratégica de serviços: operações para satisfação do cliente. São Paulo: Atlas. 1994.

HAIR JR., J. F.; BABIN, B.; MONEY, A. H.; SAMOUEL, P. Fundamentos de métodos de pesquisa em administração. Porto Alegre: Bookman, 2005.

IOMBRILLER, M. Uma investigação sobre a qualidade em serviços aplicada a companhias seguradoras. p.45-50. Dissertação (Mestrado) - Escola de Engenharia de São Carlos, Universidade de São Paulo, São Carlos, 1999.

NONAKA, I.; TAKEUCHI, H. Criação de conhecimento na empresa: como as empresas japonesas geram a dinâmica da inovação. 13.ed., Rio de Janeiro: Elsevier. 1997.

NONAKA, I.; TOYAMA, R. The knowledge-creating theory revisited: knowledge creation as a synthesizing process. Knowledge Management Research \& Practice, p.2-10. 2003.

cross ${ }^{\text {ref }}$

SANDHOF, K.. Fatores humanos no processo de desenvolvimento de software: um estudo visando qualidade, p 1830. Dissertação (Mestrado) - Escola Politécnica, Universidade de São Paulo, São Carlos, 2004.

VICENTE, S. A. Análise de aplicação dos padrões TMN no gerenciamento de sistemas de CRM. p.1-12. Dissertação (Mestrado) - Escola Politécnica, Universidade de São Paulo, São Paulo, 2003.

SVEIBY, K.E. A nova riqueza das organizações: gerenciando e avaliando patrimônios de conhecimento. Título original: The New Organization Wealth, 7.ed, Rio de Janeiro: Campus. 1998.

YIN, R. K. Estudo de caso: planejamento e métodos. São Paulo: Bookman. 2001.

\section{Claudia Andressa Cruz}

Graduação em Engenharia de Produção / UFSCar

Mestranda do Programa de Pós em Engenharia de Produção

\section{Bolsista CAPES}

Escola de Engenharia de São Carlos - Universidade de São Paulo

Av. Trabalhador São-carlense, 400 Cep: 13566-590

Tel. (16)9711-6742 e-mail: claudia.andressa@gmail.com

Edna de Almeida Rodrigues

Graduação em Administração / Centro Universitário de Araraquara

Mestranda do Programa de Pós em Engenharia de Produção

Escola de Engenharia de São Carlos - Universidade de São Paulo

Professora do Centro Universitário de Araraquara e da Faculdade Politécnica de Matão

Av. Trabalhador São-carlense, 400 Cep: 13566-590 
Tel. (16) 3373-8286 e-mail: rodrigues.pmep@ig.com.br

Marcelo Seido Nagano

Pós-Doutor em Computação Aplicada / LAC-INPE

Professor Doutor do Departamento de Engenharia de Produção

Escola de Engenharia de São Carlos - Universidade de São Paulo

Av. Trabalhador São-carlense, 400 Cep: 13566-590

Tel. (16) 3373-9380 e-mail: drnagano@usp.br

Recebido para publicação em: 19/01/07

Aceito para publicação em: 22/05/07 